\title{
The enhancement of weakly exothermic polymerization fronts
}

\author{
D. M. G. Comissiong • L. K. Gross • V. A. Volpert
}

Received: 18 November 2005 / Accepted 26 June 2006 / Published online: 15 December 2006

(C) Springer Science+Business Media B.V. 2006

\begin{abstract}
The propagation of one-dimensional waves resulting from chemical reactions in a sandwich-type two-layer setting is considered. One layer, termed the polymerization layer, contains the monomer and initiator molecules needed for the initiation of a self-propagating polymer front. The other layer will be referred to as the enhancement layer, and it contains the necessary reactants to support a highly exothermic self-propagating reaction wave. Heat exchange occurs between the layers, and as a result, there is a net diffusion of heat away from the region undergoing the more exothermic reaction. As frontal polymerization (FP) reactions are known not to be very exothermic, an overall transfer of heat from the enhancement layer into the polymerization layer takes place. An analysis of the basic state of the system is carried out to investigate the effect of heat transfer on the polymerization reaction. An enhancement layer is shown to promote FP. This analysis is applicable to the manufacture of thin polymer films by FP.
\end{abstract}

Keywords Enhancement layer $\cdot$ Frontal polymerization $\cdot$ Free-radical polymerization $\cdot$ Mathematical model Traveling wave

\section{Introduction}

Frontal polymerization (FP) refers to a chemical process whereby monomer molecules are converted to polymer chains in a spatially localized reaction zone. The self-propagating polymerization front travels through the reaction vessel leaving the polymer in its wake. The first FP experiments were performed in 1972 by Chechilo et al. [1]. FP has been subjected to intense theoretical and experimental study within recent years (see e.g. [2, 3] and the references therein).

D. M. G. Comissiong $(\varangle)$

Center for Mathematics, University of Coimbra, Apartado 3008, 3001-454 Coimbra, Portugal

e-mail:donna@mat.uc.pt

L. K. Gross

Department of Theoretical and Applied Mathematics, The University of Akron, Akron, OH 44325-4002, USA

V. A. Volpert

Department of Engineering Sciences and Applied Mathematics, Northwestern University, Evanston IL 60208-3125, USA 
FP is a more energy-efficient process than traditional polymerization techniques presently used in industry. Upon initiation of the polymer front, no additional heat source is required to propagate the front through the reaction vessel. This self-propagating front is sustained via thermal diffusion and exothermic Arrhenius reaction kinetics.

FP has the potential for producing novel materials that cannot be obtained by traditional polymerization methods. Experimentalists have used FP to produce, for example, uniform composites, hydrogels, copolymers, highly homogeneous polymer blends, and functional gradient materials [4].

The reaction mechanism in FP is similar to that of self-propagating high-temperature synthesis (SHS). In SHS, a combustion wave is generated and subsequently travels through the reaction medium, converting powdered reactants into ceramics or metallic alloys. Materials produced by SHS are highly uniform, and the procedure is simple, easy to manage, and fast. However, FP waves are significantly less exothermic than the aforementioned SHS combustion waves. As slowly propagating wave fronts are prone to instabilities (see e.g. [5]), it is therefore more challenging to produce homogeneous products via FP. Further study of FP may lead to its use in manufacturing, yielding similar advantages to those gained with SHS.

In recent years, experimentalists have achieved polymer film preparation via FP [6]. Crivello and coworkers successfully utilized FP in thin reaction zones with certain oxirane and oxetane monomers by using photoactivated initiators $[7,8]$. While in previous studies of FP, the heat produced by the initial FP reaction was sufficient for the generation of a polymer front and for its subsequent self-propagation through the reactant mixture, experimentalists have witnessed that this is often not the case for thin reaction layers. This is because of additional energy losses that are characteristic of wave propagation in this geometry. Loss of heat causes a decrease in frontal speed, quenching the reaction. It is also known that fronts which travel at a reduced speed are subject to various instabilities. Indeed, frontal instabilities are commonly associated with FP reactions. They lead to the formation of inhomogeneous polymer products.

The purpose of this study is to suggest a method for the enhancement of weakly exothermic polymerization fronts. We show that preheating the reactants causes more complete polymerization and more stable propagation of the front. With this goal in mind, we introduce a secondary reaction into the system by adding an enhancement layer to the mixture (see Fig. 1). This layer will contain the reactants necessary for initiating a highly exothermic chemical reaction front, thus acting as a supplementary heat source. The frontal polymerization reaction will be contained in a zone referred to as the polymerization layer. Our model will permit heat exchange between layers, allowing heat to be transferred into and out of the FP reaction zone. Furthermore, when the enhancement front precedes the polymerization front, heat will flow into the region directly ahead of the approaching polymer front.

We consider one-dimensional chemical wave propagation in a sandwich-type two-layer model. This particular configuration is attained via separate energy-conservation equations in each layer that are coupled through volumetric heat-transfer terms. The mathematical analysis of a similar model was outlined by Shkadinskii and Krishenik [9] in the context of combustion studies. We have utilized a generalization of this model recently to consider the effect that an inert material has on propagating polymerization fronts when heat transfer is allowed. For that case, we demonstrated the existence of multiple propagating fronts in the system when one of the layers was unreactive.

Fig. 1 System under study

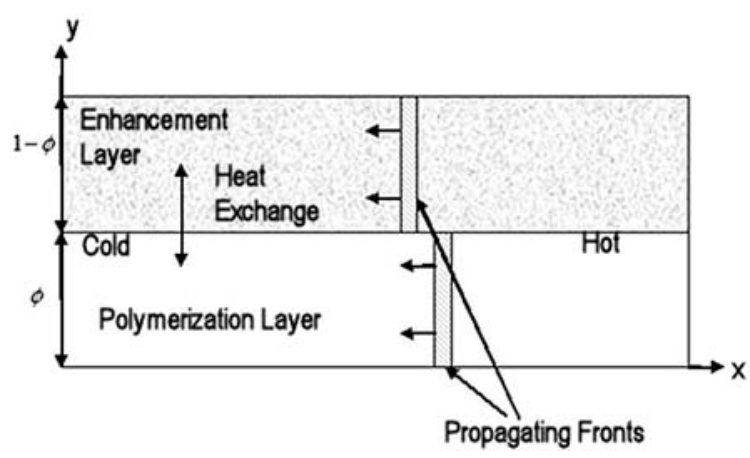


We performed a linear stability analysis of the propagating fronts in the polymerization layer, and later specified the conditions necessary for the onset of oscillatory propagation [10].

Unlike the case with an inert layer, heat is now generated by the supplementary layer. We perform an analysis of the basic state of the system, and illustrate the enhancement effect for FP.

\section{Mathematical model}

We consider two adjacent thin layers. One layer consists of a reactive mixture, which initially contains a monomer and initiator, and in which the polymerization process can occur. The other layer also contains a reactive mixture which can undergo an exothermic chemical conversion. We assume that the exothermic reaction in the second layer can be described by a simple one-step net reaction $A \rightarrow B$. Next, there is thermal contact between the two layers, and therefore there is heat exchange between the layers as illustrated by Fig. 1.

We assume that the layers are thin enough to prevent undesirable transverse variations in the polymerization front. In such cases, a one-dimensional model is appropriate. It follows that heat is conducted between layers on a time scale which is much shorter than that associated with the passage of reaction fronts within layers. We remark that providing a numerical estimate of the film thickness for which this assumption is valid would require experimental verification. While this is possible, it is beyond the scope of our current analysis.

As discussed previously, the second more exothermic reaction serves the purpose of enhancing propagation of the insufficiently exothermic polymerization wave. We will refer to the second layer as the enhancement layer, while the first layer is the polymerization layer. In order to formulate a mathematical model, we first discuss the polymerization process occurring in the first layer.

The polymerization process is the free-radical polymerization which involves a standard sequence of chemical reactions [11, Chapter 3]. The process begins when the initiator decomposes, forming two radicals. Each radical can then combine with a monomer, initiating a polymer chain. A polymer chain grows by combining with another monomer to form a longer chain, and terminates by combining with a radical, either another growing chain or an initiator radical. Thus, the kinetic scheme involves the decomposition step, initiation step, propagation step and the termination step. These kinetics equations can be simplified using the steady-state assumption. Use of this assumption has been justified in the context of a FP problem in [12]. After applying this, we obtain the following mass balance equations.

$$
\begin{aligned}
& \frac{\partial I}{\partial t}+k_{d} I=0, \\
& \frac{\partial M}{\partial t}+k_{e} \sqrt{I} M=0 .
\end{aligned}
$$

These equations account for the change in the initiator and monomer concentrations due to the chemical reactions. Here $I$ and $M$ are the concentrations of the initiator and the monomer, respectively, $t$ is the time, and $k_{d}$ and $k_{e}$ are the decomposition and the polymerization reaction-rate parameters which depend on the temperature $T_{1}$ of the polymerization layer. This dependence is given by the Arrhenius law

$k_{d}=k_{d}^{0} \exp \left\{-E_{d} /\left(R T_{1}\right)\right\}, \quad k_{e}=k_{e}^{0} \exp \left\{-E_{e} /\left(R T_{1}\right)\right\}$,

where $R$ is the gas constant, $k_{d}^{0}, k_{e}^{0}$ and $E_{d}, E_{e}$ are the frequency factors and activation energies of the two reactions.

Next, we need to formulate the energy balance in the polymerization layer. Since the main heat-producing step is the propagation step [13], the heat equation has the form

$\phi c_{1} \rho_{1} \frac{\partial T_{1}}{\partial t}=\phi \lambda_{1} \frac{\partial^{2} T_{1}}{\partial \widetilde{x}^{2}}+\phi Q_{1} \rho_{1} k_{e} \sqrt{I} M-\alpha\left(T_{1}-T_{2}\right)$.

Here $\lambda_{1}$ is the thermal conductivity, $c_{1}$ is the specific heat and $\rho_{1}$ is the mixture density. The rise in temperature induced per unit concentration of reacted monomer is

$q_{1}=Q_{1} / c_{1}$. 
Next, $T_{2}$ is the temperature of the enhancement layer, and $\phi$ is the ratio of the thickness of the polymerization layer to the total thickness of the two layers. The last term of the heat-balance equation describes heat exchange between the two layers.

The reaction process in the enhancement layer can be described by the system of two equations. The kinetic equation that represents the mass balance is written for the concentration $A$ as

$\frac{\partial A}{\partial t}+k_{a} A=0$

where

$k_{a}=k_{a}^{0} \exp \left\{-E_{a} /\left(R T_{2}\right)\right\}$,

with $k_{a}^{0}$ and $E_{a}$ being the frequency factor and the activation energy of the reaction, respectively. The heat balance in the enhancement layer has the form

$(1-\phi) c_{2} \rho_{2} \frac{\partial T_{2}}{\partial t}=(1-\phi) \lambda_{2} \frac{\partial^{2} T_{2}}{\partial \widetilde{x}^{2}}+(1-\phi) Q_{2} \rho_{2} k_{a} A-\alpha\left(T_{2}-T_{1}\right)$.

Here $\lambda_{2}$ is the thermal conductivity, $c_{2}$ is the specific heat and $\rho_{2}$ is the mixture density. The rise in temperature induced per unit concentration of reacted $A$ is

$q_{2}=Q_{2} / c_{2}$.

In a fixed coordinate frame $\widetilde{x},-\infty<\widetilde{x}<\infty$, the wave propagates along the $\widetilde{\mathrm{x}}$-axis in the direction of decreasing $\tilde{x}$. We introduce a moving coordinate system $x=\tilde{x}-\varphi(t)$ where $\varphi$ is the location of a characteristic point of the wave at time $t$. Thus, $\varphi_{t} \equiv \mathrm{d} \varphi(t) / \mathrm{d} t<0$ is the velocity of the polymerization front.

We rewrite the equations in the moving coordinate system and further simplify the problem making use of the fact that the activation energies of the decomposition and polymerization reactions are large, which results in narrow reaction zones. In the limit of infinite activation energy the reaction zone shrinks to a moving surface, termed a front. The same is true for the enhancement reaction. In this case the equations must be solved without the reaction terms both ahead of and behind the reaction fronts and matched at the reaction fronts by satisfying certain matching conditions. The reaction fronts in the two layers do not have to be situated at the same spatial location, i.e., at the same $x$. By introducing the moving coordinate system and choosing the characteristic point of the polymerization wave to be the location of the front, we fix the polymerization front at $x=0$. The enhancement front is at $x=a(t)$, which is unknown and has to be found in the course of solution of the problem. Thus, we solve the reactionless equations

$$
\begin{aligned}
& \frac{\partial I}{\partial t}-\varphi_{t} \frac{\partial I}{\partial x}=0, \\
& \frac{\partial M}{\partial t}-\varphi_{t} \frac{\partial M}{\partial x}=0, \\
& \frac{\partial T_{1}}{\partial t}-\varphi_{t} \frac{\partial T_{1}}{\partial x}=\kappa_{1} \frac{\partial^{2} T_{1}}{\partial x^{2}}-\alpha_{1}\left(T_{1}-T_{2}\right), \\
& \frac{\partial A}{\partial t}-\varphi_{t} \frac{\partial A}{\partial x}=0, \\
& \frac{\partial T_{2}}{\partial t}-\varphi_{t} \frac{\partial T_{2}}{\partial x}=\kappa_{2} \frac{\partial^{2} T_{2}}{\partial x^{2}}-\alpha_{2}\left(T_{2}-T_{1}\right)
\end{aligned}
$$

between the fronts as well as in the regions ahead of both fronts and behind them. Here

$\kappa_{1}=\frac{\lambda_{1}}{c_{1} \rho_{1}}, \quad \kappa_{2}=\frac{\lambda_{2}}{c_{2} \rho_{2}}, \quad \alpha_{1}=\frac{\alpha}{c_{1} \rho_{1} \phi}, \quad \alpha_{2}=\frac{\alpha}{c_{2} \rho_{2}(1-\phi)}$. 


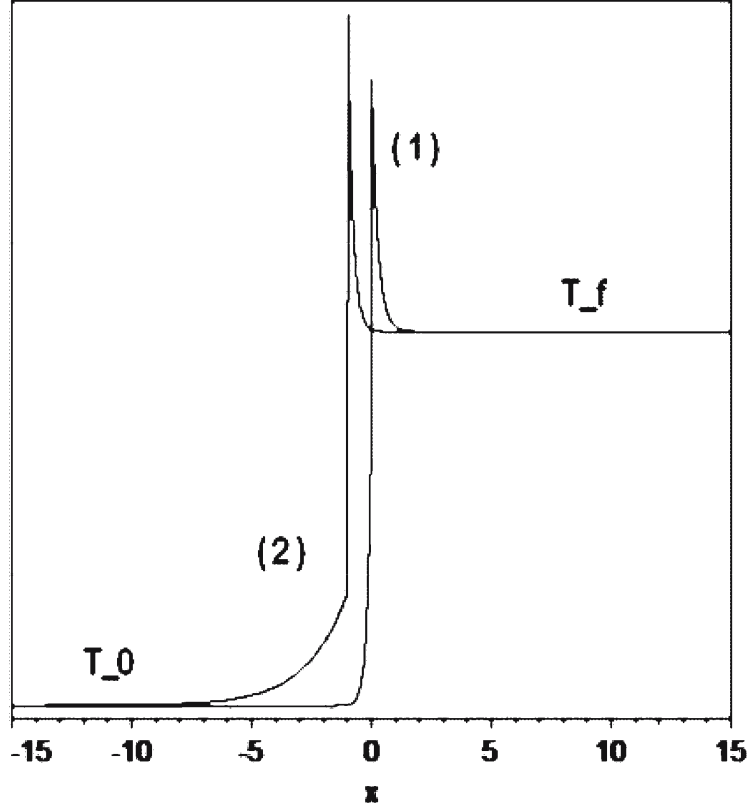

Fig. 2 Schematic of the temperature distribution in the problem. Here the polymerization front is located at $x=0$ and is labeled by (1). The enhancement front is located at $x=a<0$. In this diagram, the enhancement front (2) is shown to be more exothermic, and ahead of the polymerization front

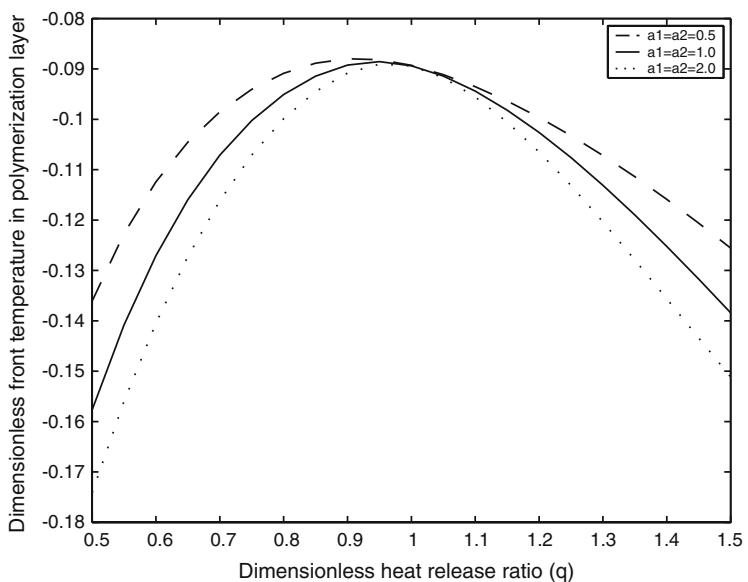

Fig. 3 Graph showing the dimensionless front temperature in the polymerization layer $\left(\theta_{1}\right)$ against the dimensionless heat release ratio $(q)$

We remark that it is not known a priori which of the two fronts goes ahead of the other, i.e., whether $a(t)$ is positive or negative. Figure 2 is an illustration of the case where $a(t)$ is negative. If $a(t)>0$, we solve the above equations for $-\infty<x<0,0<x<a(t)$ and $a(t)<x<\infty$, while in the case $a(t)<0$ the regions are $-\infty<x<a(t)$, $a(t)<x<0$, and $0<x<\infty$.

Boundary conditions far ahead of the wave describe the initial state of the layers:

$x=-\infty: \quad T_{1}=T_{2}=T_{0}, \quad M=M_{0}, \quad I=I_{0}, \quad A=A_{0}$

Here, $T_{0}$ is the initial temperature, and $I_{0}, M_{0}$ are the amount of the initiator and monomer present in the initial mixture. Far behind the wave the final state is described by

$x=+\infty: \quad T_{1}=T_{2}=T_{f}$.

This last condition states that far behind the reaction front the polymerization temperature goes to a constant value $T_{f}$, and that because of the heat exchange between the layers, the enhancement temperature attains the same value. We remark that the final temperature $T_{f}$ is unknown, and must be determined in the course of solution of the problem.

The matching conditions [10] have the form

$$
\begin{aligned}
& {\left[T_{1}\right]_{0}=0, \quad\left[T_{2}\right]_{a}=0, \quad \kappa_{1}\left[\frac{\partial T_{1}}{\partial x}\right]_{0}=q_{1}\left(M_{0}-M_{b}\right) \varphi_{t},} \\
& \kappa_{2}\left[\frac{\partial T_{2}}{\partial x}\right]_{a}=q_{2} A_{0}\left(\varphi_{t}+a_{t}\right), \\
& \varphi_{t}^{2}=F_{1}\left(T_{b 1}\right), \quad M_{b}=M_{0} \exp \left(-j_{0}\right), \quad\left(\varphi_{t}+a_{t}\right)^{2}=F_{2}\left(T_{b 2}\right) .
\end{aligned}
$$


The brackets denote a jump in a quantity across the front

$[v]_{0}=\left.v\right|_{x=0^{+}}-\left.v\right|_{x=0^{-}}, \quad[v]_{a}=\left.v\right|_{x=a(t)^{+}}-\left.v\right|_{x=a(t)^{-}}$.

Next, $T_{b 1}$ and $M_{b}$ are the temperature and monomer concentration at the polymerization front (i.e., at $x=0$ ), respectively, $T_{b 2}$ is the temperature at the enhancement front (i.e., at $x=a(t)$ ). Finally, the functions $F_{1}$ and $F_{2}$ are given by

$$
\begin{aligned}
& F_{1}\left(T_{b 1}\right)=\frac{\kappa_{1} k_{d}^{0} R T_{b 1}^{2}}{2 q_{1} M_{0} E_{d}} \exp \left(j_{0}-\frac{E_{d}}{R T_{b 1}}\right)\left(\int_{0}^{j_{0}} \frac{\mathrm{e}^{\eta}-1}{\eta} \mathrm{d} \eta\right)^{-1}, \\
& j_{0} \equiv 2 \sqrt{I_{0}} \frac{k_{e}^{0}}{k_{d}^{0}} \exp \frac{E_{d}-E_{e}}{R T_{b 1}}, \\
& F_{2}\left(T_{b 2}\right)=\frac{\kappa_{2} k_{a}^{0} R T_{b 2}^{2}}{2 q_{2} A_{0} E_{a}} \exp \left(-\frac{E_{a}}{R T_{b 2}}\right) .
\end{aligned}
$$

We remark that the form of these functions is the same as those often used in mathematical models for FP. For the details of the derivation of the functions $F_{1}$ and $F_{2}$, the reader is referred to [5] and [10].

\section{Steady-state analysis}

In this section we determine stationary solutions of the above problem, which correspond to uniformly propagating one-dimensional traveling waves in the original problem. Our primary objective is to determine the effect that the enhancement layer has on the propagating polymerization front.

We solve the following reactionless equations ahead of the fronts, behind the fronts and between the fronts

$$
\begin{aligned}
& \frac{\widehat{\mathrm{d} I}}{\mathrm{~d} x}=0, \\
& \frac{\mathrm{d} \widehat{M}}{\mathrm{~d} x}=0,
\end{aligned}
$$

$\kappa_{1} \frac{\mathrm{d}^{2} \widehat{T}_{1}}{\mathrm{~d} x^{2}}-\widehat{u} \frac{\mathrm{d} \widehat{T}_{1}}{\mathrm{~d} x}-\alpha_{1}\left(\widehat{T}_{1}-\widehat{T}_{2}\right)=0$

$\frac{\mathrm{d} \widehat{A}}{\mathrm{~d} x}=0$

$\kappa_{2} \frac{\mathrm{d}^{2} \widehat{T}_{2}}{\mathrm{~d} x^{2}}-\widehat{u} \frac{\mathrm{d} \widehat{T}_{2}}{\mathrm{~d} x}-\alpha_{2}\left(\widehat{T}_{2}-\widehat{T}_{1}\right)=0$,

subject to the boundary conditions

$$
\begin{array}{ll}
x=-\infty: & \widehat{T}_{1}=\widehat{T}_{2}=T_{0}, \quad \widehat{M}=M_{0}, \quad \widehat{I}=I_{0}, \quad \widehat{A}=A_{0}, \\
x=+\infty: & \widehat{T}_{2}=\widehat{T}_{2}=\widehat{T}_{f}
\end{array}
$$

and the matching conditions

$$
\begin{aligned}
& {\left[\widehat{T}_{1}\right]_{0}=\left[\widehat{T}_{2}\right]_{\widehat{a}}=0, \quad \kappa_{1}\left[\frac{\mathrm{d} \widehat{T}_{1}}{\mathrm{~d} x}\right]_{0}=-q_{1} \widehat{u}\left(M_{0}-\widehat{M}_{b}\right),} \\
& \kappa_{2}\left[\frac{\mathrm{d} \widehat{T}_{2}}{\mathrm{~d} x}\right]_{\widehat{a}}=-q_{2} \widehat{u} A_{0},
\end{aligned}
$$


$\left[\widehat{T}_{1}\right]_{\widehat{a}}=0, \quad\left[\widehat{T}_{2}\right]_{0}=0, \quad \kappa_{1}\left[\frac{\mathrm{d} \widehat{T}_{1}}{\mathrm{~d} x}\right]_{\widehat{a}}=0, \quad \kappa_{2}\left[\frac{\mathrm{d} \widehat{T}_{2}}{\mathrm{~d} x}\right]_{0}=0$,

$\widehat{u}^{2}=F_{1}\left(\widehat{T}_{b 1}\right), \quad \widehat{M}_{b}=M_{0} \exp \left(-\widehat{j}_{0}\right), \widehat{j}_{0} \equiv 2 \sqrt{I_{0}} \frac{k_{e}^{0}}{k_{d}^{0}} \exp \frac{E_{d}-E_{e}}{R \widehat{T}_{b 1}}$,

$\widehat{u}^{2}=F_{2}\left(\widehat{T}_{b 2}\right)$

Note that we supplemented the matching conditions from the previous section by (20) which manifest the continuity of the polymerization temperature and its derivative at the front of the enhancement reaction, and visa versa. Here the quantities with the hats denote the stationary solution, and $\widehat{u}$ is the speed of the uniformly propagating wave. The solution of the problem depends on whether $\widehat{a}$ is greater than or less than zero. Consider first the case $\widehat{a}>0$. The solution that satisfies (11)-(17) can be written in the form

$$
\begin{aligned}
& \widehat{M}(x)=\left\{\begin{array}{ll}
M_{0}, & x<0 \\
\widehat{M}_{b}, & 0<x<\widehat{a}, \\
\widehat{M}_{b}, & x \geq \widehat{a}
\end{array} \quad \widehat{I}(x)= \begin{cases}I_{0}, & x<0 \\
0, & 0<x<\widehat{a}, \\
0, & x \geq \widehat{a}\end{cases} \right. \\
& \widehat{A}(x)= \begin{cases}A_{0}, & x<0 \\
A_{0}, & 0<x<\widehat{a}, \\
0, & x \geq \widehat{a}\end{cases} \\
& \widehat{T}_{1}(x)= \begin{cases}T_{0}+C_{2} \exp \left(\lambda_{2} x\right)+C_{3} \exp \left(\lambda_{3} x\right), & x<0 \\
B_{0}+B_{1} \exp \left(\lambda_{1} x\right)+B_{2} \exp \left(\lambda_{2} x\right) & 0<x<\widehat{a}, \\
+B_{3} \exp \left(\lambda_{3} x\right), & x \geq \widehat{a}\end{cases}
\end{aligned}
$$

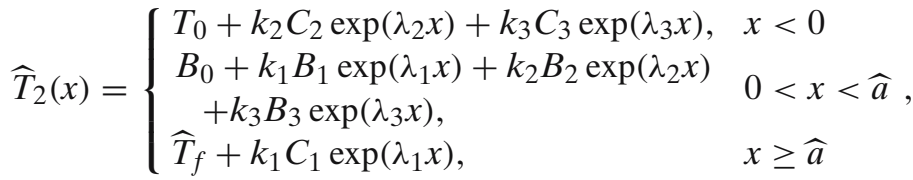

Here $\lambda_{1}, \lambda_{2}$, and $\lambda_{3}$ are the roots of the characteristic equation

$\kappa_{1} \kappa_{2} \lambda^{3}-\widehat{u}\left(\kappa_{1}+\kappa_{2}\right) \lambda^{2}+\left(\widehat{u}^{2}-\alpha_{1} \kappa_{2}-\alpha_{2} \kappa_{1}\right) \lambda+\widehat{u}\left(\alpha_{1}+\alpha_{2}\right)=0$.

It can be shown that this equation has three real roots for all $\alpha_{1}>0, \alpha_{2}>0, \kappa_{1}>0, \kappa_{2}>0, \widehat{u}>0$. Moreover, one of these roots is always negative (we denote it by $\lambda_{1}$ ), while the other two roots are always positive (we denote them by $\lambda_{2}$ and $\lambda_{3}$ ). It is convenient to write this equation in the nondimensional form as

$\kappa^{0} \nu^{3}-\left(1+\kappa^{0}\right) v^{2}+\left(1-\alpha_{i}^{0} / \kappa^{0}-\alpha_{r}^{0} \kappa^{0}\right) v+\alpha_{r}^{0}+\alpha_{i}^{0} / \kappa^{0}=0$.

Here

$\kappa^{0}=\frac{\kappa_{2}}{\kappa_{1}}, \quad \alpha_{1}^{0}=\frac{\kappa_{1}}{\widehat{u}^{2}} \alpha_{1}, \quad \alpha_{2}^{0}=\frac{\kappa_{2}}{\widehat{u}^{2}} \alpha_{2}$,

$v_{j}=\frac{\kappa_{1}}{\widehat{u}} \lambda_{j}, \quad(j=1,2,3)$

and $k_{j}$ in (25) are given by

$k_{j}=1-\frac{v_{j}^{2}-v_{j}}{\alpha_{1}^{0}}$.

Applying the matching conditions (19), (20) to the above solution for the temperatures, we determine the final temperature, $\widehat{T}_{f}$ and the constants $C_{1}, C_{2}, C_{3}$ and $B_{0}, B_{1}, B_{2}, B_{3}$. These solutions are listed in the Appendix. 
Next, we determine the propagation velocity $\widehat{u}$, the temperatures $\widehat{T}_{b 1}$ and $\widehat{T}_{b 2}$ at the reaction fronts as well as the distance $\widehat{a}$ between the fronts. The equations that relate these four quantities are

$\widehat{u}^{2}=F_{1}\left(\widehat{T}_{b 1}\right), \quad \widehat{u}^{2}=F_{2}\left(\widehat{T}_{b 2}\right)$

and

$\widehat{T}_{b 1}=T_{0}+C_{2}+C_{3}, \widehat{T}_{b 2}=\widehat{T}_{f}+k_{1} C_{1} \exp \left(\lambda_{1} \widehat{a}\right)$,

which follows from the definition of $\widehat{T}_{b 1}$ and $\widehat{T}_{b 2}$ as the temperatures at the fronts and solution (24), (25). Nondimensionalizing Eqs. 31, 32 and using

$\widehat{M}_{b}=\exp \left(-\widehat{j}_{0}\right)$,

we obtain

$$
\begin{aligned}
& \widehat{u}^{2}=u_{a 1}^{2}\left(1+\sigma_{1} \theta_{1}\right)^{2} \exp \left(\frac{Z_{e} \theta_{1}}{1+\sigma_{1} \theta_{1}}\right)\left(\widehat{j}_{0} \mathrm{e}^{-\widehat{j}_{0}} \int_{0}^{\hat{j}_{0}} \frac{\exp (\eta)-1}{\eta} \mathrm{d} \eta\right)^{-1}, \\
& \widehat{u}^{2}=u_{a 2}^{2}\left(1+\sigma_{2} \theta_{2}\right)^{2} \exp \left(\frac{Z_{a} \theta_{2}}{1+\sigma_{2} \theta_{2}}\right) \\
& \theta_{1}+1=-\frac{\alpha_{1}^{0}}{q \kappa^{0}} \frac{e_{2} v_{2}\left(v_{1}-v_{2}\right)-e_{3} \nu_{3}\left(v_{1}-v_{3}\right)}{v_{2} e_{2} e_{3}\left(v_{1}-v_{2}\right)\left(v_{1}-v_{3}\right)\left(v_{2}-v_{3}\right)}+\frac{1-\mathrm{e}^{-\hat{j}_{0}}}{v_{2} \nu_{3}\left(v_{1}-v_{2}\right)\left(v_{1}-v_{3}\right)} \\
& \times\left\{\left(1+\alpha_{1}^{0}\right)\left(v_{1}-v_{2}-v_{3}\right)-v_{1}^{2}+v_{2}^{2}+v_{3}^{2}+v_{2} v_{3}-v_{1} v_{2} v_{3}+v_{1} v_{2}\left(v_{1}-v_{2}\right)+v_{1} v_{3}\left(v_{1}-v_{3}\right)\right\}, \\
& \theta_{2}+1=-\frac{\alpha_{1}^{0}\left(\nu_{1}-v_{2}-v_{3}\right)+\nu_{1} \nu_{2} \nu_{3}-v_{2} \nu_{3}}{\kappa^{0} \nu_{2} \nu_{3}\left(v_{1}-v_{2}\right)\left(v_{1}-v_{3}\right)}+q\left(1-\mathrm{e}^{-\hat{j}_{0}}\right) \\
& \times\left(\frac{\alpha_{2}^{0}}{\alpha_{2}^{0}+\alpha_{1}^{0} \kappa^{0}}+e_{1} \frac{\left(v_{1}^{2}-v_{1}-\alpha_{1}^{0}\right)\left(1+\alpha_{1}^{0}-v_{2}-v_{3}+v_{2} \nu_{3}\right)}{v_{1} \alpha_{1}^{0}\left(v_{1}-\nu_{2}\right)\left(v_{1}-v_{3}\right)}\right) \text {. }
\end{aligned}
$$

Here $\theta_{1}$ and $\theta_{2}$ are the nondimensional temperatures at the fronts,

$\theta_{1}=\frac{\widehat{T}_{b 1}-T_{a 1}}{q_{1} M_{0}}, \quad \theta_{2}=\frac{\widehat{T}_{b 2}-T_{a 2}}{q_{2} A_{0}}$,

where $T_{a 1}=T_{0}+q_{1} M_{0}$ and $T_{a 2}=T_{0}+q_{2} A_{0}$ are the adiabatic temperatures (i.e., the final temperatures in the case that no heat exchange between the layers is allowed and conversion in the polymerization process is complete). Note that the right-hand sides of (36), (37) implicitly depend on $\widehat{u}$ through the constants $\alpha_{1}^{0}$ and $\alpha_{2}^{0}$ as well as the nondimensional characteristic roots $v$. Next, the function $\widehat{j}_{0}$ in (34), (36), which is given by (22), and which can be understood as a measure of incompleteness of the chemical conversion (see (33)), can be written in the form

$\widehat{j_{0}}=j_{*} \exp \left(-\frac{Z_{d e} \theta_{1}}{1+\sigma_{1} \theta_{1}}\right), \quad j_{*}=2 \sqrt{I_{0}} \frac{k_{e}^{0}}{k_{d}^{0}} \exp \left(\frac{E_{d}-E_{e}}{R T_{a 1}}\right)$.

Note that $\widehat{j}_{0}>j_{*}$ and the $\widehat{j}_{0}$-dependent factor in (34) goes to one as $\widehat{j}_{0} \rightarrow \infty$, while $\theta_{1} \rightarrow 0$ in this limit. As a result, $\widehat{u}$ goes to the adiabatic propagation velocity $u_{a 1}$ that occurs in case of complete conversion and is given by

$u_{a 1}^{2}=\frac{\kappa_{1} R T_{a 1}^{2}}{q_{1} M_{0} E_{d}} \sqrt{I_{0}} k_{e}^{0} \exp \left(-\frac{E_{e}}{R T_{a 1}}\right)$.

Similarly,

$u_{a 2}^{2}=\frac{\kappa_{2} R T_{a 2}^{2}}{q_{2} A_{0} E_{a}} k_{a}^{0} \exp \left(-\frac{E_{a}}{R T_{a 2}}\right)$. 
Finally,

$\sigma_{1}=\frac{q M_{0}}{T_{a 1}}, \quad \sigma_{2}=\frac{q A_{0}}{T_{a 2}}, \quad e_{j}=\mathrm{e}^{\lambda_{j} \widehat{a}}(j=1,2,3), \quad q=\frac{q_{1} M_{0}}{q_{2} A_{0}}$,

$Z_{e}=\frac{E_{e} q_{1} M_{0}}{R T_{a 1}^{2}}, \quad Z_{d e}=\frac{\left(E_{d}-E_{e}\right) q_{1} M_{0}}{R T_{a 1}^{2}}, \quad Z_{a}=\frac{E_{a} q_{2} A_{0}}{R T_{a 2}^{2}}$,

with $Z_{e}, Z_{d e}, Z_{a}$ being analogous to the Zeldovich number that is used in the combustion theory.

To determine the nondimensional front temperatures $\theta_{1}$ and $\theta_{2}$, as well as the propagation velocity $\widehat{u}$ and the distance $\widehat{a}$ between the fronts, we need to solve the system of Eqs. 34-37. The characteristic roots $v$ are solutions of (27) and depend on the parameters of the problem as well as $\widehat{u}$. The system can be reduced to a single equation for $\theta_{1}$ which we solve numerically. This is done in the following manner. First, $\widehat{u}^{2}$ is already defined in terms of $\theta_{1}$ in (34). We can use this to determine $\theta_{2}$ from (35) in terms of $\theta_{1}$. The roots $v_{i}$ can be found in terms of $\widehat{u}^{2}$ from the characteristic Eq. (27). From (37) we can find an expression for $e_{1}$ in terms of $\theta_{1}$. Finally, using

$e_{2}=\exp \left(\lambda_{2} a_{0}\right)=\left(\exp \left(\lambda_{1} a_{0}\right)\right)^{\left(\frac{\lambda_{2}}{\lambda_{1}}\right)}=e_{1}^{\left(\frac{\lambda_{2}}{\lambda_{1}}\right)} \equiv e_{1}^{\left(\frac{\nu_{2}}{\nu_{1}}\right)}$,

$e_{3}=\exp \left(\lambda_{3} a_{0}\right)=\left(\exp \left(\lambda_{1} a_{0}\right)\right)^{\left(\frac{\lambda_{3}}{\lambda_{1}}\right)}=e_{1}^{\left(\frac{\lambda_{3}}{\lambda_{1}}\right)} \equiv e_{1}^{\left(\frac{v_{3}}{v_{1}}\right)}$,

we see that (36) reduces to the aforementioned single equation for $\theta_{1}$. We are then able to determine the other unknown quantities while ensuring that $\widehat{a}>0$; this being an assumption that led to our solution.

In the case $\widehat{a}<0$ the solution procedure is similar to the one presented above. We again derive a system of four equations for $\theta_{1}, \theta_{2}, \widehat{u}$ and $\widehat{a}$. The first two equations are the same as (34), (35), while (36), (37) are replaced by

$$
\begin{aligned}
& \theta_{1}+1=\frac{\alpha_{1}^{0}}{q \kappa^{0} e_{1} \nu_{1}\left(v_{1}-v_{3}\right)\left(\nu_{1}-v_{2}\right)}-\frac{\alpha_{1}^{0}}{\kappa^{0} q \nu_{1} \nu_{2} \nu_{3}} \\
& +\left(1-\exp \left(-j_{0}\right)\right)\left(\frac{\left(v_{1}-v_{2}-v_{3}\right)\left(1+\alpha_{1}^{0}\right)-v_{1}^{2}+v_{2}^{2}+v_{3}^{2}}{v_{2} v_{3}\left(v_{1}-v_{2}\right)\left(v_{1}-v_{3}\right)}\right) \\
& \times\left(\frac{-v_{1} v_{2} v_{3}+v_{2} v_{3}+v_{1}^{2} v_{2}-v_{2}^{2} v_{1}+v_{1}^{2} v_{3}-v_{1} v_{3}^{2}}{v_{2} v_{3}\left(v_{1}-v_{2}\right)\left(v_{1}-v_{3}\right)}\right) \text {, } \\
& \theta_{2}+1=-\frac{1}{\kappa^{0}} \frac{\alpha_{1}^{0}\left(v_{1}-v_{2}-v_{3}\right)+v_{1} \nu_{2} \nu_{3}-v_{2} v_{3}}{\nu_{2} \nu_{3}\left(v_{1}-v_{2}\right)\left(v_{1}-v_{3}\right)} \\
& +e_{2}\left(\frac{\left(v_{2}^{2}-v_{2}-a_{1}\right)\left(1+a_{1}+v_{1} v_{3}-v_{1}-v_{3}\right)}{a_{1} v_{2}\left(v_{1}-v_{2}\right)\left(v_{2}-v_{3}\right)}\right)\left(1-\exp \left(-j_{0}\right)\right) \\
& -e_{3}\left(\frac{\left(v_{3}^{2}-v_{3}-a_{1}\right)\left(1+a_{1}+v_{1} v_{2}-v_{1}-v_{2}\right)}{a_{1} v_{3}\left(v_{1}-v_{3}\right)\left(v_{2}-v_{3}\right)}\right)\left(1-\exp \left(-j_{0}\right)\right) .
\end{aligned}
$$

We use the same solution method to reduce these to a single equation for $\theta_{1}$, which we solve numerically for the case $\widehat{a}<0$.

\section{Results and discussion}

Our objective is to investigate the effect the enhancement layer has on the polymerization reaction front. We will show that the polymerization front is supported by heat exchange with a more exothermic reaction.

We set the parameters for all the numerical calculations as follows

$j_{*}=1, Z_{e}=8, Z_{d e}=6, Z_{1}=8, \sigma_{1}=0 \cdot 4, \sigma_{2}=0 \cdot 4, u_{a 1}=u_{a 2}=1$. 
It is not feasible to solve the system analytically because of the large number of parameters in the model. However, by using specific values which are typical of these types of reactions, our results are indicative of a more general result. For simplicity, we make the heat diffusivities equal in both regions (i.e., $\kappa^{0}=1$ ).

To start, we define

$a_{1} \equiv \alpha_{1} \frac{\kappa_{1}}{u_{a 1}^{2}}, \quad a_{2} \equiv \alpha_{2} \frac{\kappa_{2}}{u_{a 2}^{2}}$.

As we set the adiabatic velocities $u_{a 1}=u_{a 2}=1$, and $\kappa_{1}=\kappa_{2}$, it follows whenever $a_{1}=a_{2}$, the heat exchange coefficients are equal in both the polymerization and enhancement layers (i.e., $\alpha_{1}=\alpha_{2}$ ). Upon increasing the value of $a_{1}=a_{2}$, we augment the values of the heat exchange coefficients $\alpha_{1}=\alpha_{2}$. This implies an increase in inter-layer heat transfer.

The parameter $\theta_{1}$ will also play a key role in the discussion. From the definition of $\theta_{1}$ given in (38), it follows that less negative values indicate polymerization reaction temperatures $\left(\widehat{T}_{b 1}\right)$ that are closer to the theoretical adiabatic reaction temperature $\left(T_{a 1}\right)$. This corresponds to more complete polymerization reactions, indicating that polymerization is enhanced as desired.

Consider also the dimensionless parameter $q$ given in (41). We observe that for $q<1$ the enhancement layer contains the more exothermic reaction, and for $q>1$, the polymerization reaction is the more exothermic one. With this in mind, we can refer to $q$ as the heat-release ratio. As previously stated, we expect the best results when the more exothermic reaction is contained in the enhancement layer (i.e., $q<1$ ).

To begin our investigation, we take $a_{1}=a_{2}=0 \cdot 5$, and we observe the effect that a change in the heat release ratio $q$ has on the front temperature of the polymerization layer $\theta_{1}$. In order to see the effect that a greater inter-layer heat-exchange would have on the system, we increase the values $a_{1}=a_{2}=1.0$ and further still to $a_{1}=a_{2}=2 \cdot 0$, and repeat our calculations. Figure 3 illustrates that, in general, larger heat-exchange coefficients result in more negative values of $\theta_{1}$, meaning that greater inter-layer heat exchange results in less complete polymerization reactions. We therefore focus on the lower values for the heat exchange parameters $a_{i}$, where $i=1,2$. We take the value of $q_{1}$ to be a fixed constant, and vary the value of $q_{2}$ to alter the heat exchange ratio $q$. We see from Fig. 3 that the optimal range for the heat release ratio $q$ (i.e., the range that results in the least negative values for $\theta_{1}$ ) is approximately $0.9<q<1$ for the parameters under investigation.

As such, we focus on the range $0.9<q<1$ in Fig. 4. The reaction conditions for polymerization are better in this $q$ interval because the fronts remain fairly close together. The more exothermic the reaction, the faster the corresponding velocity of the reaction front. As a result, when the enhancement reaction is much more exothermic

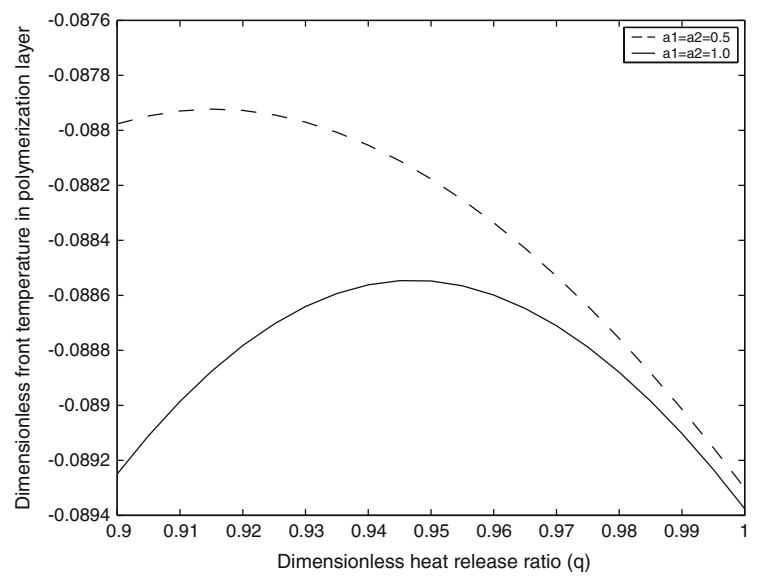

Fig. 4 Magnified view of Fig. 3 for the dimensionless front temperature in the polymerization layer $\left(\theta_{1}\right)$ against dimensionless heat release ratio in the range $0.9<q<1.0$

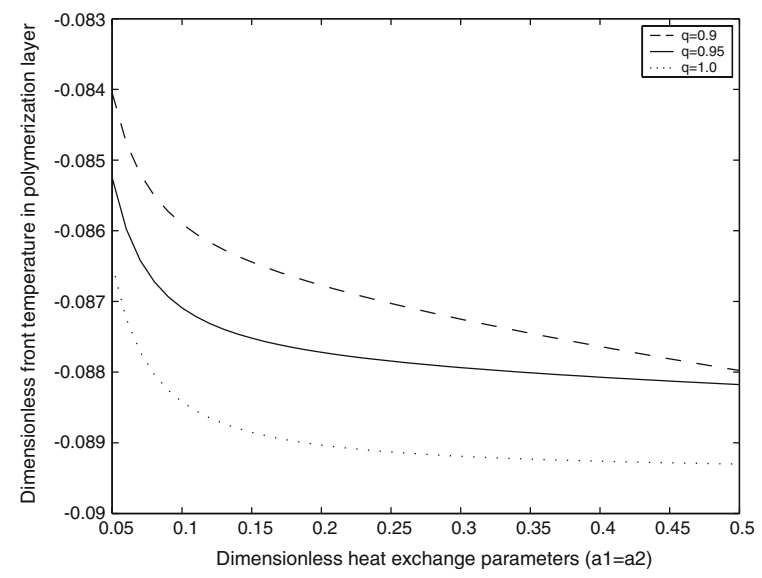

Fig. 5 Graph showing the value of the dimensionless polymerization front temperature $\left(\theta_{1}\right)$ for various values of the heat exchange parameters $a_{1}=a_{2}$. Here the heat release ratios are set to $q=0.9, q=0.95, q=1.0$ in descending order 
than the polymerization reaction or vice versa (i.e., $q \ll 1$ or $q \gg 1$ ), the fronts are too far apart for an enhancement effect to be felt in the polymerization layer. As such, we achieve best results when the enhancement reaction is more exothermic one $(q<1)$, but not too exothermic to precede the polymerization front by too large a distance. We remark that if the supplementary reaction was too exothermic, and heat exchange was allowed far away from the polymerization front, the bulk polymerization reactions could occur far away from the frontal reaction zone. However, our goal is to suggest a way to promote polymerization in the frontal mode.

We proceed to set the heat release ratio to $q=0 \cdot 9$. We then determine the polymerization front temperature $\theta_{1}$ for a spectrum of values of $a_{1}=a_{2}$. In this way we are able to observe the effect that capacity of inter-layer heat exchange has on the completeness of the polymerization reaction. Next, we repeat our calculations for heat release ratios of $q=0.95$ and $q=1.0$. The results are shown in Fig. 5. Once more we note that optimal conversion in the polymerization layer (i.e., less negative values for $\theta_{1}$ ) are achieved when the enhancement reaction is the more exothermic one $(q<1)$, and when inter-layer heat exchange is relatively small $\left(0.05<a_{i}<0.4\right)$. The smallest of the three heat release ratios $(q=0.9)$ is shown to give the best conversion rates for polymerization, but we note that at $a_{i}=0.4$ and beyond, the smallest heat release ratio $(q=0.9)$ begins to lose its advantage, as the values achieved for $\theta_{1}$ approach the curve for the fixed heat release ratio of $q=0.95$.

To illustrate this further for the same set of parameters, we also find the corresponding values of dimensionless position of the enhancement front $\widehat{a}$. We recall the the polymerization front is located at the origin $x=0$, and that both fronts propagate along the $\widetilde{x}$-axis in the direction of decreasing $\widetilde{x}$. A negative value of $\widehat{a}$ indicates that the polymerization front is behind the enhancement front whereas when $\widehat{a}>0$, the polymerization front is ahead. As heat exchange is allowed, $\widehat{a}<0$ indicates that the enhancement reaction front will be a heat source for pre-heating the reactants in the polymerization layer before the arrival of the front, which is the effect we wish to achieve. Our results are illustrated in Fig. 6 . We note that when $a_{i}$ is slightly less than 0.4 for $q=0 \cdot 9, \widehat{a}$ is small in magnitude and negative, indicating that the enhancement front is slightly ahead of the polymerization front. For $a_{i}$ greater than 0.4 , the polymerization front takes the lead as the curve begins to show that $\widehat{a}>0$. When the fronts are very close together, there is less chance that the polymerization reactants will be pre-heated prior to the arrival of the front. This is a likely reason for the loss of efficiency noted for the heat release ratio of $q=0.9$ close to and beyond $a_{i}=0.4$ in Fig. 5 .

We summarize our findings by plotting values of the polymerization layer temperature $\theta_{1}$ against the enhancement front position $\widehat{a}$ in Fig. 7. It is clear that the most complete polymerization reactions (i.e., least negative $\theta_{1}$

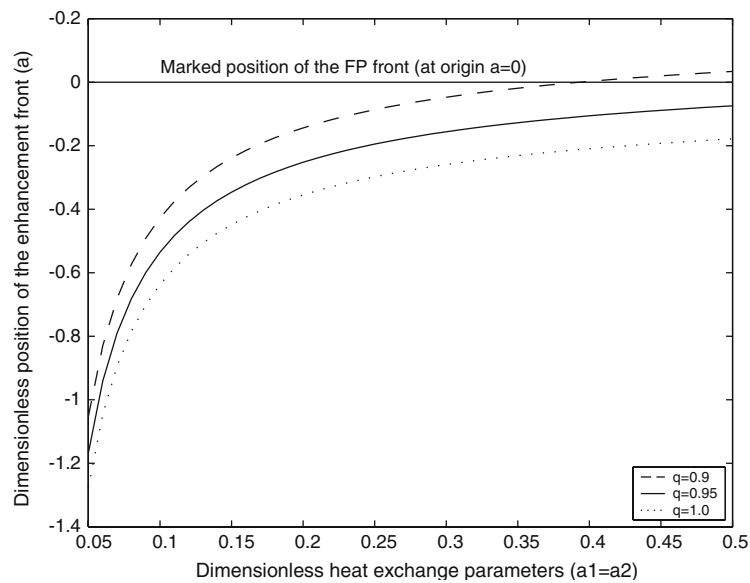

Fig. 6 Graph showing the value of the dimensionless position of the enhancement front $(a)$ for various values of the heat exchange parameters $a_{1}=a_{2}$. Here the heat release ratios are set to $q=0.9, q=0.95, q=1.0$ in descending order

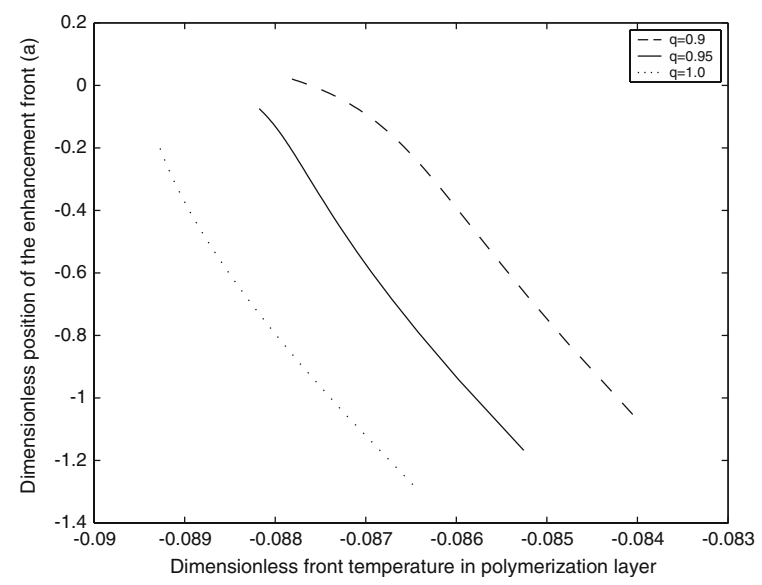

Fig. 7 Graph showing the value of the dimensionless position of the enhancement front $(\widehat{a})$ against the dimensionless front temperature in the polymerization layer $\left(\theta_{1}\right)$. Here the heat release ratios are set to $q=0.9, q=0.95, q=1.0$ 
values) are obtained when the heat release ratio $q$ is smallest (meaning that the enhancement layer contains the more exothermic reaction).

\section{Conclusion}

We have considered the steady propagation of a polymerization front in a thin layer superimposed by another layer undergoing an exothermic chemical reaction. We developed this one-dimensional two-layer sandwich model for enhancing FP reactions in thin layers. As a result of the large number of parameters in the equations, we first made the system dimensionless, and then performed an analysis of the basic state of the system. We captured the completeness of the polymerization reactions in the FP layer to quantify the enhancement effect. Specifically, we graphed trends in the dimensionless parameter $\theta_{1}$, which relates the polymerization front temperature to the theoretical adiabatic temperature of polymerization.

The enhancement effect was most pronounced when the front in the superimposed supplementary layer was ahead of the polymerization front in the lower FP layer. In this case, the overall heat is transferred from the hot product region of the superimposed enhancement layer to the preheat zone of the lower FP layer. As a result, the monomer and initiator mixture in the FP layer are heated prior to the arrival of the front, and the approaching polymerization front encounters a pre-heated reactant mixture. This ensures more complete conversion from monomer to polymer product in the FP layer. For this effect to promote FP optimally, heat exchange between the layers must be small, and the reaction in the enhancement region should be the more exothermic one. Furthermore, the separation between the reaction fronts in the two layers should not be so great as to cause bulk polymerization instead of frontal polymerization.

Acknowledgements D. M. G. Comissiong acknowledges support from the Center for Mathematics (CMUC) at the University of Coimbra, and from a Smith fellowship received at Northwestern University. This research has been supported in part by NSF grants DMS-0103856 and CTS-0138712.

\section{Appendix}

The coefficients $C_{i}, B_{i}$ and $T_{f}$ in the temperature Eqs. 24 and 25 are

$$
\begin{aligned}
C_{1}= & \left\{k_{2} v_{2}\left(k_{3}-1\right)+k_{3} v_{3}\left(1-k_{2}\right)+A\left(v_{2}\left(1-k_{3}\right)+v_{3}\left(k_{2}-1\right)\right) / e_{1}\right\} \\
& \times\left\{v_{1} v_{2}\left(k_{2}-k_{1}+k_{1} k_{3}-k_{2} k_{3}\right)+v_{2} v_{3}\left(k_{2} k_{1}+k_{3}-k_{2}-k_{3} k_{1}\right)\right. \\
& \left.+v_{1} v_{3}\left(-k_{1} k_{2}+k_{2} k_{3}-k_{3}+k_{1}\right)\right\}^{-1}, \\
C_{2}= & \left\{A\left(v_{3}\left(k_{1}-1\right)+v_{1}\left(1-k_{3}\right)\right) / e_{2}+k_{1} v_{1}\left(k_{3}-1\right)+k_{3} v_{3}\left(1-k_{1}\right)\right\} \\
& \times\left\{v_{1} v_{2}\left(k_{2}-k_{1}+k_{1} k_{3}-k_{2} k_{3}\right)+v_{2} v_{3}\left(k_{1} k_{2}+k_{3}-k_{2}-k_{3} k_{1}\right)\right. \\
& \left.+v_{1} v_{3}\left(-k_{1} k_{2}+k_{3} k_{2}-k_{3}+k_{1}\right)\right\}^{-1}, \\
C_{3}=\{ & \left\{A\left(v_{1}\left(1-k_{2}\right)+v_{2}\left(k_{1}-1\right)\right) / e_{3}+k_{2} v_{2}\left(1-k_{1}\right)+k_{1} v_{1}\left(k_{2}-1\right)\right\} \\
& \times\left\{v_{1} v_{2}\left(k_{2}-k_{1}+k_{1} k_{3}-k_{2} k_{3}\right)+v_{2} v_{3}\left(k_{1} k_{2}+k_{3}-k_{2}-k_{3} k_{1}\right)\right. \\
& \left.+v_{1} v_{3}\left(-k_{1} k_{2}+k_{3} k_{2}-k_{3}+k_{1}\right)\right\}^{-1}, \\
B_{0}=\{ & \left\{k_{1} k_{2}\left(v_{2}-v_{1}\right)\left(v_{3} t_{0}+1\right)+k_{1} k_{3}\left(v_{1}-v_{3}\right)\left(v_{2} t_{0}+1\right)+k_{3} v_{3} t_{0}\left(v_{2}-v_{1}\right)\right. \\
& \left.+k_{2} k_{3}\left(v_{3}-v_{2}\right)\left(v_{1} t_{0}+1\right)+k_{1} v_{1} t_{0}\left(v_{3}-v_{2}\right)+k_{2} v_{2} t_{0}\left(v_{1}-v_{3}\right)\right\} \\
& \times\left\{v_{1} v_{2}\left(k_{2}-k_{1}+k_{1} k_{3}-k_{2} k_{3}\right)+v_{2} v_{3}\left(k_{1} k_{2}+k_{3}-k_{2}-k_{3} k_{1}\right)\right. \\
& \left.+v_{1} v_{3}\left(-k_{1} k_{2}+k_{3} k_{2}-k_{3}+k_{1}\right)\right\}^{-1},
\end{aligned}
$$




$$
\begin{aligned}
B_{1}= & \left\{k_{3} v_{3}\left(1-k_{2}\right)+k_{2} v_{2}\left(k_{3}-1\right)\right\} \\
& \times\left\{v_{1} v_{2}\left(k_{2}-k_{1}+k_{1} k_{3}-k_{2} k_{3}\right)+v_{2} v_{3}\left(k_{1} k_{2}+k_{3}-k_{2}-k_{3} k_{1}\right)\right. \\
& \left.+v_{1} v_{3}\left(-k_{1} k_{2}+k_{3} k_{2}-k_{3}+k_{1}\right)\right\}^{-1} \\
B_{2}= & \frac{A}{e_{2}}\left(v_{3}\left(1-k_{1}\right)+v_{1}\left(k_{3}-1\right)\right) \\
& \times\left\{v_{1} v_{2}\left(k_{2}-k_{1}+k_{1} k_{3}-k_{2} k_{3}\right)+v_{2} v_{3}\left(k_{1} k_{2}+k_{3}-k_{2}-k_{3} k_{1}\right)\right. \\
& \left.+v_{1} v_{3}\left(-k_{1} k_{2}+k_{3} k_{2}-k_{3}+k_{1}\right)\right\}^{-1}, \\
B_{3}= & \frac{A}{e_{3}}\left(v_{1}\left(k_{2}-1\right)+v_{2}\left(1-k_{1}\right)\right) \\
& \times\left\{v_{1} v_{2}\left(k_{2}-k_{1}+k_{1} k_{3}-k_{2} k_{3}\right)+v_{2} v_{3}\left(k_{1} k_{2}+k_{3}-k_{2}-k_{3} k_{1}\right)\right. \\
& \left.+v_{1} v_{3}\left(-k_{1} k_{2}+k_{3} k_{2}-k_{3}+k_{1}\right)\right\}^{-1} . \\
T_{f}= & \left\{k_{2} k_{1} v_{2}+k_{1} k_{2} v_{3} v_{2} T_{0}-k_{1} v_{1} k_{2}-k_{1} k_{2} v_{3} v_{1} T_{0}-k_{3} k_{1} v_{3}\right. \\
& +k_{1} v_{1} k_{3}-k_{1} v_{2} \Lambda-k_{1} v_{2} v_{1} T_{0}-k_{1} v_{3} v_{2} T_{0} k_{3}-k_{1} k_{3} v_{2} v_{1} T_{0}+k_{1} v_{3} \Lambda \\
& +k_{1} v_{3} v_{1} T_{0}+k_{3} v_{3} k_{2}+k_{3} v_{3} v_{1} T_{0} k_{2}+k_{2} v_{2} v_{1} T_{0}-k_{2} v_{3} v_{2} T_{0} \\
& -k_{2} v_{2} k_{3}+v_{1} k_{2} \Lambda-k_{3} k_{2} v_{2} v_{1} T_{0}-k_{2} v_{3} \Lambda+k_{3} v_{2} \Lambda+v_{3} v_{2} T_{0} k_{3} \\
& \left.-k_{3} v_{3} v_{1} T_{0}-\Lambda v_{1} k_{3}\right\} *\left\{k_{2} v_{3} k_{1} v_{2}+k_{2} v_{2} v_{1}-k_{1} v_{2} v_{1}\right. \\
& -k_{1} v_{1} k_{2} v_{3}+k_{3} v_{3} v_{1} k_{2}+k_{4} v_{2} v_{1} k_{3}-k_{3} v_{3} v_{1}+k_{3} v_{3} v_{2} \\
& \left.-k_{2} v_{3} v_{2}-k_{3} v_{3} k_{1} v_{2}+k_{1} v_{1} v_{3}-k_{2} v_{2} v_{1} k_{3}\right\}^{-1},
\end{aligned}
$$

where we take

$\Lambda=\frac{\kappa_{1}}{\kappa_{2}} q_{2} M_{20}$.

\section{References}

1. Chechilo NM, Khvilivitskii RJ, Enikolopyan NS (1972) On the phenomenon of polymerization reaction spreading. Dokl Akad Nauk SSSR 204:1180-1181

2. Perry MF, Volpert VA (2004) Self-propagating free-radical binary frontal polymerization. J Engng Math 49:359-372

3. Comissiong DMG, Gross LK, Volpert VA (2005) Nonlinear dynamics of frontal polymerization with autoacceleration. J Engng Math 53:59-78

4. Washington RP, Steinbock O (2003) Frontal free-radical polymerization applications to materials synthesis. Polymer News 28:303-310

5. Schult DA, Volpert VA (1999) Linear stability analysis of thermal free-radical polymerization waves. Int J SHS 8:417-440

6. Mariani A, Fiori S (2002) Recent developments in frontal polymerizaion. Polymer Preprints 43:814-815

7. Crivello JV, Falk B, Zonca MR Jr (2004) Photoinduced cationic ring-opening frontal polymerizations of oxetanes and oxiranes. J Poly Sci Part A Polym Chem 42:1630-1646

8. Falk B, Zonca MR Jr, Crivello JV (2005) Photoactivated cationic frontal polymerization. Macromol Symp 226:97-107

9. Shkadinskii KG, Krishenik PM (1985) Steady combustion in a mixture of fuel and inert material. Combust Shock Expl Waves 21:176-180

10. Comissiong DMG, Gross LK, Volpert VA (in press) Frontal polymerization in the presence of an inert material. J Eng Math

11. Odian GG (1991) Principles of polymerization, 3rd edn. Wiley-Interscience, New York

12. Spade CA, Volpert VA (2000) On the steady state approximation in thermal free radical frontal polymerization. Chem Eng Sci 55:641-654

13. Manelis GB, Smirnov LP, Peregudov NI (1977) Nonisothermal kinetics of polymerization processes. Finite cylindrical reactor. Combust Expl Shock Wave 13:389-393 